\title{
La Physiocratie : gouvernementalité et rationalisation de l'action publique
}

\author{
Bernard Herencia \\ Université Paris-Est / Largotec (EA 4688) \\ et Université Montpellier III / CRISES (EA 4424)
}

\section{Résumé}

L'interrogation sur la nature et les finalités de l'action publique est récurrente depuis I'apparition même de la science économique au XVIIle siècle. Cet article explore la problématique de l'intervention de l'État dans le champ économique à partir des concepts de gouvernementalité et de technologies de pouvoir (Foucault et Lascoumes) dans l'œuvre du plus politique des disciples de François Quesnay : Paul Pierre Lemercier de la Rivière.

(c) 2013 IDMP/Lavoisier SAS. Tous droits réservés

Mots clés : Histoire de la pensée, physiocratie, gouvernementalité.

\section{Abstract}

Physiocracy: governmentality and rationalization of the public action. The questioning of the nature and aims of public action is recurrent since the very foundation of economics in the eighteenth century. This article explores the issue of state intervention in the economic field with the concepts of governmentality and technologies of power (Foucault and Lascoumes) in the work of the disciple the more political of Francois Quesnay: Paul Pierre Lemercier de la Rivière.

(c) 2013 IDMP/Lavoisier SAS. Tous droits réservés

Keywords: History of thought, physiocrats, governmentality.

\section{Introduction}

Dans la seconde moitié du XVIII ${ }^{\mathrm{e}}$ siècle, un groupe d'intellectuels se constitue autour de François Quesnay (1694-1774) et fonde la première véritable école économique : la physiocratie. Dans ce groupe, Paul Pierre Lemercier de la Rivière (1719-1801) développe tout particulièrement le volet politique des travaux. Ses expériences de juriste

*Auteur correspondant : bernard.herencia@univ-mlv.fr doi:10.3166/pmp.30.75-87 @ 2013 IDMP/Lavoisier SAS. Tous droits réservés 
et d'administrateur colonial nourrissent ses réflexions et son activité de publiciste des années soixante aux années quatre-vingt-dix. Son apport peut être réinterprété en termes de gouvernementalité à travers l'analyse qu'il produit des instruments d'action politique et de leurs usages. Le recours au concept introduit par Foucault est ainsi fructueux pour étudier le lien entre l'économique et le politique dans son œuvre (1). La physiocratie, dans sa construction du «gouvernement économique » ou du politique, équivalent ici (Faccarello, 1993 : 149), pose comme principe fondamental la liberté de commerce et de circulation des grains.

Ce principe n'est pas neuf en lui-même mais ce sont les physiocrates qui vont en faire un fondement politique de la société. La liberté économique est un faisceau de libertés spécifiques (liberté de consommation, de circulation des grains, du commerce extérieur, du trafic des colonies notamment), capable de stimuler la production et, avec elle, le souverain détient un puissant levier de commande. La recherche, par les physiocrates, de la meilleure efficience possible de l'articulation économique/politique les conduit à rechercher une rationalisation en matière économique (2) comme en matière politique (3) et à dégager un ensemble d'instruments pour conduire l'action politique (4).

La logique mercantiliste ${ }^{1}$ antérieure est alors inversée par les physiocrates : pour les premiers le marché est sous le contrôle d'un politique arbitraire - dédié à la puissance du prince - tandis que les seconds réclament une puissance publique sous l'emprise d'un ordre naturel dont la liberté du marché est une des expressions - pour l'intérêt commun du gouvernant et des gouvernés - . Les champs politique et économique sont alors imbriqués de telle manière qu'aucun n'a de véritable ascendant sur l'autre, leur articulation est totale : les réformes économiques attendues supposent l'édification d'un ordre politique qui en retour permet à la sphère économique de produire les meilleurs effets pour le « royaume agricole » de France.

\section{Une nouvelle raison gouvernementale}

La volonté des physiocrates de fonder une nouvelle raison gouvernementale peut être éclairée à partir des travaux de Charles, Kaplan et Napoli sur la police et de Foucault sur la gouvernementalité.

La police recouvre l'ensemble des moyens permettant d'accroître les forces de l'État en y maintenant le bon ordre, c'est-à-dire tout ce qui permet d'intégrer les activités humaines dans l'État dont le point de vue, à l'époque, est celui de la puissance, i.e. de la capacité à faire la guerre. Celui-ci doit en retour stimuler ces activités avec le souci du nombre des hommes, des nécessités de la vie, de la santé, des activités des hommes et de la circulation de leurs productions. D'une manière générale, la police doit établir l'équilibre entre les positions des vendeurs et des acheteurs et maintenir une harmonie entre intérêt privé et intérêt général (Charles, 1999 : 70). Pour Napoli (2003 : 39 et 50), c'est dès le XVII ${ }^{\mathrm{e}}$ siècle qu'émerge la question majeure du repérage du problème de l'État et de la mise en

\footnotetext{
${ }^{1}$ Le mercantilisme désigne la convergence d'auteurs européens qui, du XVle au XVIII ${ }^{\mathrm{e}}$ siècle, affirment que seule la puissance publique incarne l'intérêt national, qu'elle doit défendre des ambitions et agissements des autres Etats, notamment en matière économique par des politiques d'intervention et de protection.
} 
place « d'une rationalité gouvernementale » avec une police qui « enveloppe tout l'espace de la politique ». L'État, dès lors, par le cadre réglementaire qu'il développe, marque de son empreinte tous les rapports sociaux (Kaplan, 1986 : 25). La police désigne alors « la conduite de la chose publique dans son ensemble » et, étant liée aux événements historiques, constitue « une condition d'existence interne » de la société (Napoli, 2003 : 25-26). À l'époque du mercantilisme, elle doit consister « à conduire l'homme à la plus parfaite félicité dont il puisse jouir en cette vie » (Delamare, 1705 : 2). Au milieu du XVIII ${ }^{\mathrm{e}}$ siècle, l'émergence de l'idée d'administration fait reculer la sphère de la police pour la réduire progressivement à son activité juridictionnelle. Et, si les activités économiques répondent à des processus naturels (c'est un des fondements de l'analyse physiocratique), rien ne sert a priori de tenter de réglementer. La forme de gouvernementalité qui convient à l'État a pour principe fondamental de respecter ces processus « ou en tout cas d'en tenir compte, de les faire jouer ou de jouer avec eux » (Foucault, 2004a : 360). Ainsi, l'économie politique doit respecter une exigence d'autolimitation de la raison gouvernementale, qui repose sur la connaissance du cours naturel des choses mais ce principe n'est en aucun cas exclusif de l'action gouvernementale.

La gouvernementalité (Foucault, 2004a : 111, 321-333 et 400) est un ensemble d'institutions et de procédures, d'analyses et de réflexions, de calculs et de tactiques permettant d'exercer un pouvoir dont la principale cible est la population. Son étude renvoie à celle $\mathrm{du}$ processus de rationalisation et de technicisation de l'exercice du pouvoir. Il s'agit d'articuler les appareils spécifiques du gouvernement et les systèmes de connaissance. La fiscalité d'une part et l'instruction d'autre part constituent dans la physiocratie les cas typiques des uns et des autres. Dès lors, exercer le pouvoir politique, ce n'est plus conquérir par la force et assujettir les vaincus au tribut fiscal, mais stimuler et produire pour le bien de tous, monarque y compris. Dans ce cadre, l'impôt et l'éducation sont des instruments physiocratiques d'action politique, des dispositifs techniques et sociaux qui structurent les rapports entre la puissance publique et les citoyens, entre gouvernants et gouvernés, selon la terminologie de Lemercier de la Rivière. Globalement, le mouvement physiocratique produit alors une nouvelle raison gouvernementale fondée sur la logique « du moindre État qui émancipe «d'une mise en forme et d'une disciplinarisation par l'État » mercantiliste» (Foucault, 2004b : 31 et 34). Chez Foucault ce discours ne fonctionne pas par rupture car il ne s'insurge pas «contre l'immoralité de l'exercice du pouvoir » mercantiliste, il en adopte au contraire le langage et parfois en approche l'esprit. C'est ce que relève Steiner (2002 : 458), pour qui Quesnay, alors qu'il contribue à l'Encyclopédie (à l'époque de la guerre contre l'Angleterre), a pour objectif d'indiquer comment la politique économique doit être conduite pour accroître la richesse, améliorer le rendement fiscal et ainsi renforcer la puissance militaire.

La perception de la puissance publique dans l'analyse physiocratique est, sur ces bases, généralement associée à une volonté de libéralisation. Le libéralisme, pour Foucault (2004a : 818) est « un système soucieux du respect des sujets de droit et de la liberté d'initiative des individus » qui se caractérise par une critique de « la gouvernementalité excessive » : « on gouverne toujours trop ». Rappelons, pour compléter, la démonstration de Polanyi : le marché commence à exister dès que l'État cesse d'intervenir pour en réguler les mouvements, tout en persistant dans sa mission régalienne sans laquelle le marché n'est que jungle. Ces approches diffèrent de celle de Hayek qui entend le 
libéralisme comme un objectif à atteindre comprenant le retrait de l'État de l'économie. Dans le premier cas (Foucault et Polanyi), le libéralisme est un processus d'extension des libertés, ou ce qui revient au même, de retrait de l'intervention publique de la sphère économique, dès lors que la puissance publique conserve un rôle d'encadrement général du marché ; dans le second cas (Hayek), le libéralisme est vu comme résultat à atteindre, un horizon constitué par une sphère économique libérée de toute intervention étatique. Hayek exprime la volonté de donner naissance à un libéralisme intégral, donc distinct du moins d'État recherché par les physiocrates, caractérisé par la disparition de toute forme de coercition ; ce qui nous éloigne définitivement des physiocrates qui entendent assigner à l'État un rôle majeur.

Pour eux, le libéralisme est agissant (par recul de l'État) mais ne tend pas à l'effacement intégral de l'action gouvernementale. Le « moindre État » auquel ils aspirent, s'élève tout d'abord contre la multiplicité des polices économiques mercantilistes. Il s'agit de s'en remettre au marché pour optimiser l'allocation des ressources, des grains au premier chef. Dans cette perspective, le marché libéré (physiocratique) est conçu comme alternative au marché sous contrôle (mercantiliste) ou encore, pour employer la terminologie de Foucault, les physiocrates recherchent une disciplinarisation du marché par un État produisant de moindres polices. L'administration de l'État va à la fois s'affirmer comme « structure de travail bureaucratique » et comme « puissance gouvernementale soumise à des règles financières ». Administrer c'est, historiquement, à la fois juger, calculer, gérer, diriger, et la police en elle-même est un processus de rationalisation. Ce dernier est ainsi déjà à l'œuvre à l'époque des physiocrates et le changement qu'ils introduisent réside dans la disciplinarisation de la puissance publique et l'affirmation de nouvelles finalités pour les technologies de pouvoir par la libéralisation du commerce et la rationalisation de la puissance publique.

\section{La libéralisation économique}

En matière économique, pour les physiocrates, la rationalisation est d'abord une question de libéralisation du marché et leurs travaux affirment l'importance du droit de propriété et la nécessité d'échanger (notamment internationalement) sans intervention de l'État. La liberté de circulation n'est pas réclamée pour obtenir le grain au plus bas prix possible par la mise en concurrence des producteurs mais parce que, à leur sens, la libéralisation du commerce des grains possèdent deux vertus fondamentales : 1 / l'exportation permet de faire sortir les grains du royaume lorsque les récoltes sont trop abondantes - pour éviter qu'il ne tombent en non-valeur - de manière à maintenir les prix ; 2/ la stabilisation des prix à la hausse empêche les producteurs de s'enrichir les années de mauvaises récoltes. Dès lors, une rupture est recherchée avec les pratiques interventionnistes conçues pour maintenir artificiellement le bas niveau des prix (notamment du pain), de manière à assurer la paix sociale conformément au « pacte nourricier $»^{2}$ liant le

\footnotetext{
${ }^{2}$ Ce pacte traduit une conception organiciste (et paternaliste) de la société qui conduit le souverain à devoir non pas nourrir son peuple mais « faire en sorte qu'il soit nourri » (Kaplan, 1986: 25) en contrepartie de sa soumission.
} 
prince à ses sujets, et qui restreignent les possibilités de développement économique et l'expansion démographique. C'est l'atteinte de ces deux objectifs que les physiocrates confient à la liberté de circulation. Ils font le choix d'une décentralisation des décisions (par les acteurs économiques et non le prince) pour obtenir un «bon prix » du grain préservé des oscillations dévastatrices habituelles. L'individu dépasse la simple soumission au monarque, il devient acteur de « processus qu'il faut gérer dans ce qu'ils ont de naturel et à partir de ce qu'ils ont de naturel ». Le libéralisme est alors entendu comme liberté de circulation et fonde une « technologie de pouvoir » basée sur la «normalisation » des règles de droit (Foucault, 2004a : 49-50 et 58).

La volonté politique de changement économique peut s'exprimer à plusieurs niveaux (Samuels, $2002: 1$ ) : de la police des grains à une réflexion d'ensemble pour une régulation macroéconomique. Lorsqu'il délimite ces niveaux, Lemercier de la Rivière révèle une démarche différente de celle, par exemple, saluée par Turgot dans son Eloge à Vincent de Gournay (Schelle : 596-597). Turgot recense les éléments factuels qui permettent d' " embrasser dans toute son étendue et suivre dans ses révolutions continuelles l'état des productions naturelles, de l'industrie, de la population, des richesses, des finances, des besoins et des caprices même de la mode chez toutes les nations que le commerce réunit » car il s'agit d' «étudier le commerce en négociant, ce n'est encore qu'une partie de la science du commerce », c'est son socle. "Mais découvrir les causes et les effets cachés de cette multitude de révolutions (...) c'est l'envisager en philosophe et en homme d'État ». C'est à ce niveau supérieur que Lemercier de la Rivière (en 1767) spécule et tente d'embrasser toute la cohérence socio-économique du monde : il s'agit d'aménager la rationalisation de l'État avec le souci d'améliorer la lisibilité et la maîtrise des opérations économiques. Lemercier de la Rivière (1765 : 144) s'inspire évidemment du maître Quesnay et lorsqu'il invoque la recherche d'un « grand revenu » et d'une « grande population », il affirme qu'il « faut provoquer [je souligne] dans l'État la plus grande abondance possible » : l'action gouvernementale s'impose donc dans le principe.

Cette volonté de rationalisation s'appuie ainsi sur l'élaboration d'instruments scientifiques d'analyse économique, dont le Tableau économique de Quesnay est l'expression la plus éclatante. Charles et Steiner (1999 : 140) montrent que le problème politique est d'abord, chez les physiocrates, une question d'expertise administrative. À cette époque, l'appareil d'État et l'administration centrale s'étoffent (l'augmentation des personnels ministériels en est l'expression la plus visible). Ces institutions connaissent une évolution décisive avec la substitution des rapports de rivalité et de subordination aux relations amicales et personnelles entre les grands serviteurs de l'État (Maurepas et Boulant, 1996 : 53). Ce besoin d'experts amène Necker, lorsqu'il poursuit l'œuvre entreprise par Turgot en matière de clarification de la comptabilité publique, et Castries, en charge de la Marine, à trouver en Lemercier de la Rivière le « technicien tout désigné » (May, 1975 : 110-111) pour mener à bien la réforme de la comptabilité des Colonies et celle des Ports et Arsenaux en 1780.

Sa carrière administrative témoigne effectivement de cette expertise, mais le physiocrate est conscient que les dossiers doivent être contextualisés et qu'il est nécessaire de prendre de la hauteur. La difficulté de ce travail ne lui échappe pas, notamment lorsqu'il s'agit de l'évaluation des grandeurs macro-économiques : "le revenu général d'une nation est, dit-on, le montant, la masse de tous les revenus particuliers. Je veux bien 
admettre cette définition, pourvu que dans le calcul ou la formation de cette masse, on évite les doubles emplois. En effet, il est une multitude de particuliers dont les revenus ne doivent point être comptés dans cette masse ; et de ce nombre sont tous ceux dont les revenus se trouvent n'être que des portions prises dans d'autres revenus ${ }^{3}$. Cet extrait, préfigurant les principes de la comptabilité nationale, exprime la volonté de rationaliser les études économiques et Lemercier de la Rivière, dans sa recherche d'instruments pour optimiser l'art de gouverner, s'efforce à de nombreuses reprises de préciser les notions et de conceptualiser ses éléments de réflexion, ce qui le conduit à proposer des outils économiques tout à fait modernes ${ }^{4}$. Dans la citation précédente il approche de près l'idée de valeur ajoutée.

Il a également d'autres intuitions importantes, par exemple il met parfois en avant la consommation (et donc la demande) et son rôle moteur dans l'activité économique : « la consommation est la mesure nécessaire de la reproduction » (ibid. : 54). Lemercier de la Rivière (1792, II : 250-251) formule également l'idée d'un multiplicateur : l'accroissement du revenu national, implique une hausse de celui des cultivateurs, qui peuvent alors élever le niveau de leurs investissements ; ce qui ne peut manquer de générer une augmentation seconde du revenu. C'est l'époque durant laquelle Lemercier de la Rivière oriente ses recherches vers un plan d'action publique fondé sur des règles de conduite allant souvent à l'encontre de la doxa physiocratique : fixation autoritaire des prix, contrôle des activités stratégiques (meunerie), prohibition à l'exportation (farine), attribution de primes à la production (riz, racines et pommes de terre), aide à l'investissement ( «améliorations des terres » et défrichements), soutien à la création d'emplois manufacturiers (femmes et enfants) pour améliorer les revenus des ménages, enfin, établissement de statistiques nationales (population et terres à blé) de manière à former des plans annuels de production.

À ce stade d'évolution de sa pensée, Lemercier de la Rivière s'éloigne donc assez radicalement et évolue vers ce que nous appellerions un « dirigisme $»^{5}$. Ces derniers efforts de

\footnotetext{
${ }^{3}$ Lettre sur les économistes dans Lemercier de la Rivière, 2011 : 76.

${ }^{4}$ Cette modernité est encore attestée lorsque Lemercier de la Rivière $\left(1763, f^{\circ} 23\right)$ préconise une approche des flux en termes macroéconomiques : "On confond sans cesse les véritables intérêts du commerce qui sont ceux de l'état, avec les intérêts particuliers et momentanés de quelques marchands, objets qui ne sont rien dans l'état. Cette confusion d'idées et d'objets jette dans de grandes erreurs ; il est aisé de n'y pas tomber, il ne s'agit que de simplifier les choses en regardant comme une seule et unique personne, comme un seul et unique individu cette multitude de marchands emploiés par l'état à faciliter les consommations de ses denrées et à les faire devenir accroissement de richesses par la conversion en argent d'une de ces mêmes denrées portées chez l'étranger. Dès lors on ne calculera plus le produit particulier d'une opération particulière ; l'argent placé dans la main gauche ou dans la main droite ne paroittra plus faire une différence essentielle, à raison seulement de la place qu'il occupe, on ne fera attention qu'à son employ; on ne considérera que la masse entière de ses opérations, et le produit total de cette masse, comme le résultat unique qui puisse intéresser les richesses de l'état. »

${ }^{5}$ Lemercier de la Rivière adapte ses positions au contexte révolutionnaire. II retrouve l'essentiel de ses principes constitutionnels dans la constitution de 1791 mais les ruptures, notamment marquées par la fuite du roi et la fusillade du champ de Mars, amorcent l'éloignement de la Révolution de la monarchie constitutionnelle. Au moment de la parution de sa dernière publication (L'Heureuse Nation) l'Europe coalisée déclare la guerre à la France et la Patrie est déclarée « en danger » : Lemercier de la Rivière donne à son dernier ouvrage la forme d'un essai utopique : ce qui, probablement, lui permettra d'échapper à la Terreur.
} 
rationalisation, en dépit de leur nature peu libérale ${ }^{6}$, témoignent à nouveau de la volonté de Lemercier de la Rivière de travailler sur les instruments pour construire une gouvernementalité physiocratique, de manière à répondre encore au programme de recherche ouvert dans les années cinquante par Quesnay même si la distance s'accroît entre ses positions d'alors et la doctrine fondée par le docteur. Au titre d'instruments, il faut encore citer les Sociétés d'agriculture (avec des correspondants précieux pour les physiocrates), le Bureau de l'agriculture dirigé par Bertin ou encore la volonté récurrente de cadastrer ${ }^{7}$ la France.

Le XVIII ${ }^{\mathrm{e}}$ siècle (avec les physiocrates) fait ainsi passer la réflexion économique d'une logique de l'harmonie et de la stabilité à une logique de progrès et de croissance. Pour les philosophes, la rationalisation de l'appareil d'État traduit un progrès de la liberté parce qu'elle " garantit l'indépendance de l'individu face aux corps constitués » et crée «l'avènement de l'ordre transcendantal de la Raison » (Steinhauer, $1997: 210$ ). Pour reprendre le mot de Tocqueville (1856 : 259), l'État des physiocrates « n'a pas uniquement à commander à la nation, mais à la façonner » (Samuels, $2002: 3-4)$, tandis que « la croyance dans le progrès spontané nous rend nécessairement aveugle au rôle de l'État dans la vie économique. Ce rôle consiste souvent à modifier le rythme du changement, en l'accélérant ou en le ralentissant, selon les cas » (Polanyi, 1944 : 64). Avant l'analyse physiocratique, il est communément admis que l'État est le garant des conditions économiques permettant de régler les problèmes des subsistances et celui de l'enrichissement/puissance du prince. Avec la tentative physiocratique, la liberté du commerce des grains (en isolant la pensée des dernières années de Lemercier de la Rivière) est érigée en un instrument d'action gouvernementale capable d'atteindre simultanément ces deux objectifs. C'est une césure historique qui ouvre le débat (et il ne se refermera plus) sur le rôle économique de l'État.

\section{La rationalisation politique}

La volonté de rationaliser l'architecture gouvernementale elle-même marque la spécificité du travail de Lemercier de la Rivière qui a développé et enrichi la conception politique physiocratique jusqu'au début de la Révolution, notamment avec ses propositions pour un contrôle constitutionnel (cf. encadré). La libéralisation économique ne peut être réduite au simple recul de la présence et de l'action de l'État, au contraire il faut un État disciplinarisé et propre à produire les polices incontestables nécessaires au bon fonctionnement du marché. Le traité de Delamare, que cite fréquemment Lemercier de la Rivière, procède du mouvement général de rationalisation dans l'administration royale auquel aspire l'Economiste : il s'agit d'inventorier, de classer et de catégoriser pour consolider l'organisation de l'action publique (Lascoumes, 2004). Lorsqu'il œuvre à la codification de la législation coloniale dans les années quatre-vingt, Lemercier de la Rivière suit une logique similaire.

\footnotetext{
${ }^{6}$ Mais il s'agit alors de composer entre d'une part les Enragés (Lemercier de la Rivière appartient alors à la section des Gravilliers, comme Jacques Roux) et les Hébertistes (les Exagérés) et, d'autre part, les Girondins.

${ }^{7}$ Sur la question du cadastre, cf. Alimento (2008) et Touzery (2007).
} 


\section{Le cadre conceptuel : ordre naturel et despotisme légal}

Pour les physiocrates l'ordre social réel n'est pas l'ordre naturel, ce dernier n'est qu'une potentialité à réaliser. La mission première de la puissance publique est d'instituer l'ordre supérieur de la nature. L'homme doit en comprendre le fonctionnement et en devenir l'acteur (d'où l'importance de l'instruction publique).

À l'époque des Lumières, la loi devient une valeur fondamentale du droit : elle organise la puissance publique et structure le social. Les physiocrates exacerbent l'idée : la loi doit imposer son despotisme. Pour Lemercier de la Rivière (1767 : 72-74) la question du droit est concomitante à celle de la société. Il dissocie alors la loi positive de son fondement (sa « lettre » de sa « raison»). La raison de la loi est sa nécessité, imposée par la nature, et Lemercier de la Rivière recherche ses articulations avec le droit positif et formule les bases d'un système normatif des lois. La hiérarchisation correspondante structure dans le même temps le législatif : les lois positives résultent de l'ordre naturel ; la puissance publique les valide (elle appose son « sceau »); elles s'imposent à tous, monarque compris. Cela pacifie les relations sociales et si Lemercier de la Rivière insiste pour signifier qu'il ne s'agit que d'énoncer des lois faites par la nature (c'est leur raison), il multiplie les indications sur le soin à apporter à leur formulation (leur lettre) pour les rendre compréhensibles à tous et incontestables. Il développe alors une téléologie du droit positif : son élaboration est la mission la plus fondamentale de la puissance publique de manière que l'action politique, dont le but est l'abondance matérielle, soit la plus efficace possible en la rendant conforme à l'ordre de la nature.

C'est sur ces bases que Lemercier de la Rivière (en 1767) fonde le « despotisme légal » qu'il faut entendre comme l'empire des lois naturelles sur la société humaine par la médiation d'un monarque lui-même assujetti à cet ordre supérieur. Le despote légal est éclairé par l'évidence (de l'ordre naturel) et impose la nécessité de rationaliser la puissance publique. Il a un intérêt propre au succès de sa politique : un attribut important de ce monarque réside dans sa co-propriété sur le produit net des terres et non du sol lui-même, car une co-propriété des terres aliénerait les propriétés privées, alimenterait la dés-incitation à l'investissement et finalement rognerait le produit net à attendre de l'activité économique. La thèse de la co-propriété du produit net est un fondement essentiel du politique : les intérêts du despote légal sont confondus avec ceux de tous les acteurs. Il ne peut qu'aspirer à les satisfaire car il y est personnellement intéressé, d'autant plus que sa couronne est héréditaire. Cet intérêt commun fonde le lien social, soude la communauté nationale et place la problématique économique au cœur du politique.

À la fin de sa vie, Lemercier de la Rivière ${ }^{8}$ travaille à la rédaction d'un texte constitutionnel pour figer la structuration de la puissance publique. Il insiste tout particulièrement sur la nécessité de mettre en place un contrôle de constitutionalité pour parachever la rationalisation de la puissance publique garantissant sa conformité avec ce que les physiocrates supposent être l'ordre naturel. En effet, à défaut d'un tel dispositif de contrôle, la prétendue conformité du droit positif à l'ordre de la nature porterait en elle un germe d'arbitraire.

${ }^{8}$ Les Vœux d'un François et Essais sur les maximes et loix fondamentales (Lemercier de la Rivière, 2011). 
C'est encore par une référence à la rationalisation que Mirabeau résume le projet physiocratique : l'impôt (avec sa nature et son assiette) et la liberté du commerce, « ces deux points (...) obligent nécessairement les auteurs des mémoires à remonter à la source des dépenses et à établir les principes de la science (...) économique » (Mirabeau, 1772 : 59-60). C'est la voie qu'emprunte Lemercier de la Rivière (1767 : 25, 28, 38-39) lorsqu'il définit l'ordre naturel : c'est «l'accord parfait des moyens physiques dont la nature a fait choix pour produire nécessairement les effets physiques qu'elle attend de leurs concours ». Il réduit cette nécessité à des problématiques élémentaires : n’est-il pas «physiquement impossible de vivre sans subsistance ?»; « les hommes se multipliant» n'est-il pas nécessaire de les «multiplier par la culture ? » La finalité affichée du projet est « le bonheur et la multiplication des hommes » et ce qu'il appelle «ordre essentiel » est « un enchaînement de moyens sans lesquels il est impossible de remplir » cette finalité ; il est « l'accord parfait des institutions sociales sans lesquelles ce bonheur et cette multiplication ne pourroient avoir lieu ». Les efforts de rationalisation sont finalisés et le projet est véritablement politique : « une Société Politique est une multitude plus ou moins nombreuse d'hommes qui désirant d'augmenter leur bonheur par des secours mutuels, se réunissent et s'associent les uns aux autres par des conventions servant à régler les Droits dont ils jouiront et les devoirs qu'ils auront à accomplir » (Lemercier de la Rivière, 1792, II : 368-369). Dès lors, Lemercier de la Rivière fonde toutes ses recherches sur les moyens d'édifier un droit positif pérenne conforme à l'ordre naturel.

La vision physiocratique est souvent interprétée comme statique et conservatrice voire réactionnaire ou encore comme monarchiste voire absolutiste. Pourtant le projet de Lemercier de la Rivière (1767 : 117) ne cherche pas à légitimer l'existant, il propose une vision de ce que devrait être l'ordre politique : « je ne jette les yeux sur aucune nation, sur aucun siècle en particulier : je cherche à peindre les choses telles qu'elles doivent être essentiellement, sans consulter ce qu'elles sont ou ce qu'elles ont été, dans quelque pays que ce soit ». Il n'est pas question d'un retour à un certain âge d'or social, ni même de conserver un ordre existant parfait. Il y a, dans cette conception du monde, une volonté de construire une société fidèle à un ordre naturel, dont les règles sont supposées préexister mais dont la société réelle correspondante n'existe pas : il est nécessaire d'en fonder les principes positifs et d'établir les institutions nécessaires.

Cette finalité n'est pas frappée de déterminisme car il ne s'agit pas de laisser agir la nature : l'homme doit appréhender les règles de l'ordre et mettre en œuvre une volonté politique pour les appliquer et construire le sentier permettant à la société de s'acheminer vers ces buts ultimes. Les physiocrates cherchent à préserver leur dispositif politique de l'arbitraire et Mirabeau (1760:27) contrecarre, par avance, la « route de la servitude » de Hayek : le prince doit suivre «la route du devoir » en «tournant [les sujets] vers la vertu pratique, en les excitant au travail ». Dans ce sens, le gouvernement doit être réformé pour répondre à «l'ordre naturel et essentiel des sociétés politiques ». Lemercier de la Rivière souhaite l'avènement et non une quelconque « restauration du pouvoir absolu de l'ordre de la nature ». Avec les physiocrates, il renonce à toute démarche révolutionnaire et opte pour la raison et la science qui doivent contribuer à ce que les lois naturelles soient déclarées et portées à la connaissance de tous (souverain et sujets) de sorte qu'ils ne puissent résister à leur évidence. Hutchinson (1988 : 274, 280 et 284) observe que si Quesnay et Mirabeau débutent avec les recherches des principes agricoles et économiques, Lemercier de la Rivière se concentre d'emblée sur l'étude des fondamentaux politiques et sur la loi. 
À tout prendre la démarche politique physiocratique relève d'une volonté d'action. Au-delà de sa portée théorique, L'Ordre naturel et essentiel des sociétés politiques est un texte de circonstance dont la vocation est de répondre aux questions du temps, en particulier par des solutions aux difficultés politiques liées à une crise financière active de longue date, par la recherche d'une « construction politique » pour dynamiser l'économie du royaume et stabiliser son régime politique. Ces réflexions sur la rationalisation de l'appareil gouvernemental induisent une analyse de ses instruments d'action.

\section{Les instruments de l'action politique}

La nature de ces instruments peut être éclairée par les travaux des sciences de gestion (Lascoumes, 2004, § 12-17 et 21 ; Lascoumes et Le Galès, 2004 : introduction). à partir de leurs trois composantes : une philosophie gestionnaire, une représentation schématique de leur organisation et un substrat technique. Ces instruments sont au service d'une démarche politico-technique de résolution de problèmes. Dans ces études, l'instrument est défini comme « un dispositif à la fois technique et social qui organise des rapports sociaux spécifiques entre la puissance publique et ses destinataires en fonction des représentations et des significations dont il est porteur » (Lascoumes, 2004, § 12). La principale limite qui se présente tient à l'absence de spécificité des instruments : ils ne produisent pas que les seuls effets pour lesquels ils sont destinés par la puissance publique mais produisent des effets originaux parfois inattendus, comme les édits de 1763-64 sur la liberté de circulation des grains, par exemple, qui vont provoquer des pénuries, des flambées de prix et finalement des émeutes. Enfin, par sa triple constitution, chacun de ces instruments est une forme condensée de gouvernementalité en renvoyant à une théorisation du rapport entre gouvernants et gouvernés. C'est dans cette perspective que ces travaux peuvent être appliqués à l'approche physiocratique de manière à faire apparaître les rapports politiques et leur légitimité associés aux instruments de l'action gouvernementale.

Globalement, les fonctions étatiques telles que les synthétise Lemercier de la Rivière sont les suivantes : assurer le respect du droit naturel, c'est-à-dire le droit de propriété et la liberté économique ; percevoir l'impôt ; pourvoir aux équipements collectifs nécessaires à l'activité économique ; organiser l'instruction publique et veiller à la liberté de la presse pour diffuser la connaissance de l'ordre naturel ; représenter la Nation à l'extérieur. Ces missions permettent de construire la typologie suivante :

\begin{tabular}{|c|c|c|}
\hline $\begin{array}{c}\text { Type } \\
\text { d'instrument }\end{array}$ & Type de rapport politique & Type de légitimité \\
\hline $\begin{array}{c}\text { Législatif et } \\
\text { réglementaire }\end{array}$ & $\begin{array}{c}\text { État organisateur } \\
\text { d'une société conforme } \\
\text { à l'ordre naturel }\end{array}$ & $\begin{array}{c}\text { Les gouvernants doivent } \\
\text { permettre aux gouvernés } \\
\text { d'agir dans un cadre conforme } \\
\text { à l'ordre naturel }\end{array}$ \\
\hline Économique & $\begin{array}{c}\text { État (par le truchement } \\
\text { du monarque) co-propriétaire } \\
\text { du produit net }\end{array}$ & $\begin{array}{c}\text { Stabilité du régime politique (le } \\
\text { monarque est intéressé } \\
\text { à la performance économique } \\
\text { de la société) }\end{array}$ \\
\hline
\end{tabular}




\begin{tabular}{|c|c|c|}
\hline Fiscal & $\begin{array}{c}\text { État producteur de sûreté et } \\
\text { d'infrastructures }\end{array}$ & $\begin{array}{c}\text { Recherche d'une utilité } \\
\text { collective et d'une efficacité } \\
\text { sociale } \\
\text { t économique }\end{array}$ \\
\hline Éducatif & $\begin{array}{c}\text { État diffuseur de la } \\
\text { connaissance de l'ordre } \\
\text { naturel }\end{array}$ & $\begin{array}{c}\text { Accès de tous à l'évidence } \\
\text { de l'ordre naturel } \\
\text { Explicitation des décisions } \\
\text { des gouvernants et } \\
\text { responsabilisation des } \\
\text { gouvernés }\end{array}$ \\
\hline Publiciste & $\begin{array}{c}\text { État gardien de la liberté de la } \\
\text { presse }\end{array}$ & $\begin{array}{c}\text { Accès de tous à l'évidence } \\
\text { de l'ordre naturel } \\
\text { Acceptation des décisions } \\
\text { des gouvernants et } \\
\text { responsabilisation des } \\
\text { gouvernés }\end{array}$ \\
\hline $\begin{array}{c}\text { Normes et } \\
\text { standards }\end{array}$ & $\begin{array}{c}\text { Ajustements compétitifs au } \\
\text { sein de la société (mécanismes } \\
\text { concurrentiels) }\end{array}$ & $\begin{array}{c}\text { Supériorité des mécanismes } \\
\text { de marché libérés. }\end{array}$ \\
\hline
\end{tabular}

Cette série de six types d'instruments permet de synthétiser les axes fondamentaux de la rationalisation économique et politique par la disciplinarisation de l'appareil d'État que recherchent les physiocrates. Dans une approche générale, ces instruments laissent apparaître une régulation physiocratique, une régulation despotique-légale ${ }^{9}$ en recourant à une définition large d'une régulation comme ensemble des règles de conduite et des moyens matériels et techniques mis en œuvre par l'État pour acheminer la structuration politique de la société vers l'idéal de l'ordre naturel (et non maintenir la constance d'un tel ordre puisqu'il n'est pas inscrit dans la réalité sociale). Cette régulation despotique-légale dépend du recours aux instruments du gouvernement que nous venons de sérier. Cet usage fonde une gouvernementalité despotique-légale associée à un ensemble de technologies de pouvoir spécifiques qui en assurant le développement de l'économie politique construit un « nouveau système scientifique » (Hirschman, $1986: 21)$, une « gouvernementalité physiocratique ».

\section{Conclusion}

Finalement, la pensée des physiocrates est au carrefour de deux démarches intellectuelles, l'une en partie conservatrice (pour la stabilité du régime politique monarchique), l'autre progressiste (avec la volonté de réorganiser l'exercice du pouvoir). Dès lors, l'action des hommes, et à travers eux du politique, est indispensable, notamment pour instituer le droit positif nécessaire et l'organisation politique conforme à l'ordre naturel, «le monde alors va de lui-même » à condition pour Lemercier de la Rivière qu'il existe trois institutions

\footnotetext{
${ }^{9}$ Et non une « régulation despotique » au sens indiqué par Gaudemar, c'est-à-dire assujettie à la volonté du despote lui-même (Steiner, 1984, passim).
} 
sociales essentielles : l'institution des lois (avec les magistrats), celle de l'autorité tutélaire (le monarque) et celle de l'instruction (et de la presse) (Lemercier de la Rivière, 1767 : 447).

Plus généralement, un des objectifs de la philosophie du XVIII ${ }^{\mathrm{e}}$ siècle est d'éradiquer ou au moins de limiter l'aliénation politique des hommes. Tout individu s'inscrit dans un pacte social qui l'unit aux autres hommes. Un tel contrat engage à respecter les lois et à mettre en place les autorités nécessaires à leur sauvegarde. Cet aspect est incontestablement un des objectifs des physiocrates et cela permet de les intégrer au mouvement général des Lumières : comme elles, en dépit d'oppositions fortes avec Montesquieu ou Voltaire, ils recherchent une transformation politique et annoncent la révolution à venir. Dans cette démarche, Lemercier de la Rivière ne cherche finalement pas à savoir si politique et économique sont articulés, il recherche comment faire jouer leur indémêlable imbrication pour optimiser le fonctionnement social et ouvrir la voie à la maximisation du produit net pour assurer le bonheur des hommes et leur multiplication.

\section{Remerciements}

L'auteur remercie les réviseurs pour leurs précieuses remarques. 


\section{Bibliographie}

ALIMENTO A., (2008). Réformes fiscales et crises politiques, Lang, Bruxelles.

CHARLES L., (1999). La Liberté du commerce des grains et l'économie politique française (1750-1770), Thèse pour le doctorat en sciences économique, Université Paris I.

CHARLES L., STEINER P., (1999). Entre Montesquieu et Rousseau ,Études Rousseau 11, 83-159.

DELAMARE N., (1705). Traité de la police, Tome I, Cot, Paris.

FACCARELLO G., (1993). Les Economies politiques des Lumières, In BÉRAUD A. et FACCARELLO G., Nouvelle Histoire de la pensée économique, Tome I, La Découverte, Paris, 141-153.

FOUCAULT M., (2004a). Sécurité, territoire, population, Gallimard, Paris.

FOUCAUlT M., (2004b). Naissance de la biopolitique, Gallimard, Paris.

HIRSCHMAN A. O., (1986). Vers une Économie politique élargie, Minuit, Paris.

HUTCHINSON T., (1988). Before Adam Smith, Blackwell, Oxford.

KAPLAN S. L., (1986). Le Pain, le peuple et le Roi. La Bataille du libéralisme sous Louis XV, Perrin, Paris.

LASCOUMES P., (2004). La Gouvernementalité, Le Portique 13-14.

LASCOUMES P., LE GALÈS P. et al., (2004). Gouverner par les instruments, Presses de Sciences Po, Paris.

LEMERCIER DE LA RIVIÈRE P. P., (1763). Mémoire pour Monseigneur le duc de Choiseul, servant de réponse aux motifs de mon Rappel et à plusieurs de ses dernières dépêches qui ne me sont parvenues que pendant le cours de ma maladie dit Mémoire justificatif. Archives nationales Col. E276, pièce 43, 85 p.

LEMERCIER DE LA RIVIÈRE P. P., (1765), Observations sur le mémoire intitulé Réflexions d'un citoyen, Journal de l'agriculture du commerce et des finances, novembre 1765, Tome III, Paris, Knapen, 100-146.

LEMERCIER DE LA RIVIÈRE P. P., (1767). L'Ordre naturel et essentiel des sociétés politiques, Nourse, Londres.

LEMERCIER DE LA RIVIÈRE P. P., (1792). L'Heureuse Nation, Buisson, Paris.

LEMERCIER DE LA RIVIÈRE P. P., (2011). Canevas d'un code constitutionnel. Euvres politiques (17871789), Présentation et transcription : Bernard Herencia, Genève, Slatkine.

MAUREPAS A. de et BOULANT A., (1996). Les Ministres et les ministères du siècle des Lumières, Christian, Paris.

MAY, L.-P., (1975). Le Mercier de la Rivière, CNRS, Paris.

MIRABEAU V. R. de, (1760). Théorie de l'impôt, Scientia verlag, Aalen.

MIRABEAU V. R. de, (1772). Ébauche d'un essai sur les principes généraux du gouvernement (1772), In SIRÉN O., Kina och den kinesiska tanken I sverige på 1700-talet, Lychnos, 1948, Uppsala, 54-65.

NAPOLI P., (2003). Naissance de la police moderne, La Découverte, Paris.

POLANYI K., (1944). La Grande Transformation, Gallimard, Paris.

QUESNAY F., (2005). Euvres économiques complètes, INED, Paris.

SAMUELS W. J., (2002). Economics, Governance and Law, Elgar, Cheltenham.

SCHELLE G., (1913). Euvres de Turgot, Tome I, Alcan, Paris.

STEINER P., (1984). Régulation despotique ou despotisme légal ? Revue d'économie politique 2, 301-308.

STEINER P., (2002). Quesnay, In GREFFE et al., Dictionnaire des grandes æuvres économiques, Dalloz, Paris, 457-463.

STEINHAUER A., (1997). Le Parti physiocratique, Thèse de science politique, Paris I.

TOCQUEVILLE A. de, (1856). L’Ancien Régime et la révolution (1856), Flammarion, Paris.

TOUZERY M., (dir.) (2007). De l'Estime au cadastre en Europe, XIII'-XVIII siècles. II. L'Époque moderne. Actes du colloque tenu à Bercy les 4-5 décembre 2003, Paris, Comité pour 1'histoire économique et financière de la France. 
